\title{
RELAÇÕES DE CAUSALIDADE ENTRE O RETORNO DAS AÇÕES DO SETOR DE CONSTRUÇÃO CIVIL BRASILEIRO E INDICADORES MACROECONÔMICOS
}

\author{
Marcelo de Lima Balzana Filho ${ }^{1}$ \\ Ricardo Bordeaux-Rego ${ }^{2}$
}

Resumo: Este artigo teve o objetivo de analisar se há uma relação de causalidade entre as ações do setor de construção civil brasileiro e indicadores macroeconômicos. Foram utilizados teste de raiz unitária, teste de causalidade de Granger e análise de regressão multivariada para tentar modelar relações de retornos entre indicadores defasados e as ações. Utilizando sete ações e oito indicadores, o estudo concluiu: CDI causa uma ação, INCC causa quatro ações, IGPM causa duas ações, IBOV causa cinco ações, PIB causa três ações e a taxa de desemprego causa quatro. $O$ único indicador econômico que é causado pelas ações é o PIB, sendo este causado por BISA3 e PDGR3. Tentou-se construir modelos multivariados para prever o retorno das ações através dos dados defasados dos indicadores, mas esses modelos não tiveram sucesso em obter resultados próximos dos retornos reais no período avaliado.

Palavras-chave: Mercado de Ações, Ações da Construção Civil, Indicadores Macroeconômicos, Causalidade de Granger.

Abstract: This article analyzed whether there is a causal relationship between the stocks of the Brazilian construction industry and macroeconomic indicators. It used unit root tests, Granger causality tests and multivariate regression analysis to build a model showing the relation between lagged indicators and the stock returns. Using seven stocks and eight indicators, the study concluded that CDI cause one stock, INCC cause four stocks, IGPM cause two stocks, IBOV cause five stocks, PIB cause three stocks and unemployment rate cause four stocks. The only economic indicator that is caused by the shares is the GDP, which is caused by BISA3 and PDGR3. The study tried to build multivariate regression models to predict stock returns using data lagged indicators, but these models have not been successful in obtaining results close to the real returns on the studied period.

Key-words: Brazilian Stock Market, Brazilian Construction Sector, Macroeconomic Indicators, Granger Causality.

\footnotetext{
1 Dept $^{\mathbf{o}}$ de Engenharia de Produção, Universidade Federal Fluminense Campus Niterói. E-mail: marcelobalzana@hotmai.com

2 Dept $^{\mathbf{o}}$ de Engenharia de Produção, Universidade Federal Fluminense Campus Niterói. E-mail: ribordeaux@hotmail.com
} 


\section{INTRODUÇÃO}

A partir do trabalho de Balzana Filho et al.(2014), onde foi estudada a influência das ações do ramo imobiliário com os indicadores econômicos aprofunda-se a análise, e são realizados testes de causalidade para descobrir se algum indicador é capaz de anteceder temporalmente o movimento de alguma ação do setor de construção civil brasileiro, ou o contrário.

Destaca-se que foi escolhido estudar este setor pela atenção que está sendo dada ao mercado imobiliário, em virtude da recente alta no preço dos imóveis em quase todas as grandes cidades brasileiras. Embora os preços dos imóveis estejam subindo nos últimos anos, percebe-se que o mesmo movimento não acontece com a maioria dos retornos das ações do setor de construção civil no mesmo período. Ressalta-se também que o mercado imobiliário está em expansão desde 2004, alcançando um PIB setorial de até 11,6\% no ano de 2010. Este setor sofreu e ainda sofre grande influência de alguns programas governamentais como $\mathrm{O}$ "Minha Casa, Minha Vida", o incentivo à facilidade de obtenção do crédito baseada em um modelo de expansão do consumo. Além disso, recebeu investimentos voltados para a realização dos eventos da Copa do Mundo de 2014 e outros direcionados a cidades específicas como as Olimpíadas de 2016, no Rio de Janeiro.

\section{REVISÃO DA LITERATURA}

\subsection{Revisão de Trabalhos}

Em Lee (1992), são investigadas interações e relações de causalidade entre os retornos das ações do mercado norte americano com a taxa livre de risco, atividade industrial e inflação no período de janeiro de 1947 até dezembro 1987. O objetivo era verificar a validade dos modelos que apontavam uma correlação negativa entre a inflação e o retorno dos ativos no mercado. Foi utilizada a técnica de análise estatística VAR, que são vetores auto-regressivos, e foi constatado: retornos de ações e a atividade industrial possuem uma relação positiva; retornos de ações e inflação não possuem nenhuma causalidade entre si e não foi achada uma correlação negativa com significância estatística; a inflação responde negativamente a mudanças nos retornos reais das taxas livres de risco; a inflação possui pouca influencia na atividade industrial, mas sua correlação é negativa; retorno das ações parece explicar uma parte da atividade industrial, positivamente. A principal conclusão deste artigo foi que não há causalidade entre as ações e a inflação, sendo esses resultados compatíveis com as hipóteses de Fama para as relações negativas entre inflação e mercado de ações.

$\mathrm{O}$ artigo de Perales e Robins (2002) estuda as relações entre o mercado financeiro e a atividade real no México, no período de janeiro de 1990 a maio de 2000. O estudo usa a causalidade de Granger em dados mensais para descobrir se a Bolsa Mexicana de Valores causa ou é causada pela inflação, produção industrial, base monetária, taxa de desemprego, taxa de juros, taxa de câmbio, Índice Dow Jones e taxa de juros trimestral dos títulos do tesouro norte americano. As evidências encontradas são de que a volatilidade da inflação causa variações na atividade real e que a base monetária tem grande influencia na inflação e na produção industrial. Existe uma relação causal simultânea entre a inflação e a base monetária, e uma relação causal tanto dos títulos do tesouro americano quanto do Índice Dow Jones com a Bolsa Mexicana. Os autores concluem que há uma grande influência do ciclo econômico norte americano no mercado financeiro mexicano.

No artigo de Nunes et al. (2005), realiza-se uma análise para a relação das variáveis macroeconômicas com o mercado de ações brasileiro, no período de janeiro de 1995 a dezembro de 2004, que compreende o momento pós implantação do Plano Real. Foram usadas como variáveis relevantes para o estudo: o PIB, o Índice Bovespa, a inflação medida pelo IGP-M, a taxa de juros, o 
câmbio e o spread do C-bond. Os resultados do trabalho apontam para a constatação de que o Índice Bovespa possui uma relação negativa com a inflação, a qual não demonstrou uma relação negativa com a atividade econômica. Entretanto, foi verificada uma antecedência no movimento do mercado de ações em relação às variações da inflação (NUNES et al., 2005, p. 587).

$\mathrm{O}$ artigo de Gan et al. (2006) estuda quais as relações entre o Índice de Ações da Nova Zelândia e sete indicadores macroeconômicos, no período de janeiro de 1990 a janeiro de 2003. Os autores usam testes de cointegração e causalidade de Granger em suas análises e as variáveis usadas no trabalho são: inflação, taxa de câmbio, produção industrial, base monetária, taxa de juros de curto e longo prazo e preço do barril de petróleo. A análise concluiu que o Índice Neozeolandês é influenciado negativamente pela inflação, sendo as alterações nesta variável alcançando seu máximo de influencia com um atraso de quatro meses. Além da inflação, a atividade industrial se mostrou uma variável com influencia positiva e o destaque negativo ficou com a base monetária e a taxa de juros de longo prazo. $\mathrm{O}$ artigo termina ressaltando que o mercado da Nova Zelândia ainda é pequeno e por isso pode estar mais exposto a indicadores externos ao país, sugere-se estudos complementares.

Singh (2010) estuda as relações das variáveis: mercado Indiano, inflação, produção industrial e taxa de câmbio. Fazendo uso de uma base de dados mensal entre abril de 1995 e março de 2009, Singh procurou entender melhor quais são as correlações entre as quatro variáveis selecionadas e que tipo de relações causais essas variáveis têm entre si. Os resultados mostraram que todos os indicadores possuem correlações positivas altas, excetuando a taxa de câmbio que possui uma correlação positiva baixa com as outras variáveis. $\mathrm{O}$ Teste de Causalidade de Granger concluiu o seguinte: o índice de ações causa variações na atividade industrial e na inflação, somente a atividade industrial causa o índice; a taxa de câmbio se mostrou independente das outra variáveis, tanto no sentido de causar e ser causada. $\mathrm{O}$ trabalho conclui que o mercado de ações indiano ainda tem poucos investidores e operadores, com a consequência de que as relações observadas neste trabalho não sejam "conclusivas" para a economia da Índia, a qual ainda precisa se desenvolver mais.

\section{METODOLOGIA}

\subsection{Teste de Raiz Unitária de Dickey-Fuller Aumentado}

Este trabalho utilizou, da mesma forma que Balzana Filho et. al (2014), o teste de raiz unitária de Dickey-Fuller Aumentado (ADF), que é derivado do teste de Dickey-Fuller (DF), apresentado em Dickey e Fuller (1979). Faz-se necessário utilizar o teste ADF para testar a hipótese nula das séries temporais serem não-estacionárias, pois é requisito para o Teste de Causalidade de Granger que as séries sejam estacionárias.

Optou-se por não utilizar testes de cointegração devido a todas variáveis independentes terem sido consideradas estacionárias, conforme tabela 4 apresentada adiante. Logo, as séries utilizadas no estudo não cumprem as premissas necessárias ao teste de cointegração: variáveis não-estacionárias e integradas de mesma ordem. Ou seja, testar-se-ia a possibilidade de existir uma série estacionária entre duas séries nãoestacionárias que deveriam necessariamente ser estacionárias após o mesmo número de diferenciação.

\subsection{Teste de Causalidade de Granger}

Apresentado em Granger (1969), o teste de causalidade de Granger serve para medir se uma variável $X$ é capaz de Granger-causar alterações em uma variável Y. Usa-se o termo "Grangercausar", porque esse modelo causal não é relativo ao sentido endógeno de causalidade, e sim ao sentindo de precedência temporal que a variável $\mathrm{X}$ 
pode ter em relação a variável Y. Baseiase na ideia de que acontecimentos futuros não podem explicar acontecimentos presentes ou passados, somente acontecimentos passados podem causar eventos presentes ou futuros.

Supondo duas variáveis temporais, $\mathrm{X}$ e $\mathrm{Y}$, estacionárias, é possível dizer que a variável $\mathrm{X}$ causa $\mathrm{Y}$ se os valores defasados de $\mathrm{X}$ tem alguma influência no valor presente de $\mathrm{Y}$, na presença dos valores defasados de Y. Do mesmo modo, diz-se que $\mathrm{Y}$ causa $\mathrm{X}$ se os valores atrasados de $\mathrm{Y}$ podem ajudar a explicar o valor atual de $\mathrm{X}$, mesmo considerando valores defasados de $\mathrm{X}$. Para testar tais hipóteses são usadas as seguintes equações:

$$
\begin{aligned}
& X_{t}=\sum_{j=1}^{m} a_{j} X_{t-j}+\sum_{j=1}^{m} b_{j} Y_{t-j}+\varepsilon_{t} \\
& Y_{t}=\sum_{j=1}^{m} c_{j} X_{t-j}+\sum_{j=1}^{m} d_{j} Y_{t-j}+\eta_{t}
\end{aligned}
$$

Onde $^{\varepsilon_{t}}$ e $\eta_{t}$ são variáveis aleatórias independentes e identicamente distribuídas, sendo $\varepsilon_{t} \sim N\left(0, \sigma_{1}^{2}\right) \quad$ e $\eta_{t} \sim N\left(0, \sigma_{2}^{2}\right), \quad$ ambas sem autocorrelação.

É utilizado o teste F para verificar a hipótese nula do somatório dos coeficientes serem estatisticamente iguais à zero. Usando a fórmula:

$$
F=\frac{\left(S Q E_{R}-S Q E_{I R}\right) / m}{S Q E_{I R} /(n-2 m-1)}
$$

Onde $S Q E_{R}$ é a soma dos quadrados dos resíduos da regressão restrita, que é o modelo formado somente pelos termos defasados da variável dependente; $S Q E_{I R}$ é a soma dos quadrados dos resíduos da regressão irrestrita, que se refere ao modelo composto tanto pelos termos defasados da variável dependente quanto da outra variável; $m$ é o número de defasagens testadas; $n$ é o número de observações.

Podem ocorrer então três possíveis casos de causalidade (CARNEIRO, 1997, p. 4):
1. Causalidade unilateral: Quando $\sum b_{j} \neq 0$, na primeira equação, e $\sum c_{j}=0$, na segunda equação, ocorre causalidade de $Y$ em relação a $X$, mas não há causalidade de $\mathrm{X}$ para $\mathrm{Y}$. $\mathrm{O}$ inverso, que é a causalidade unilateral de $\mathrm{X}$ para Y, ocorre se $\sum b_{j}=0$ e $\sum c_{j} \neq 0$ nas primeira e segunda equações, respectivamente.

2. Retroalimentação ou Bicausalidade: Acontece quando $X$ causa $\mathrm{Y}$ e $\mathrm{Y}$ causa $\mathrm{X}$, ocorrendo através dos resultados $\sum b_{j} \neq 0$ e $\sum c_{j} \neq 0$, nas duas regressões.

3. Independência: No caso de não haver causalidade dos termos defasados de $\mathrm{X}$ para com $\mathrm{Y}$ e nem causalidade dos coeficientes atrasados de $\mathrm{Y}$ para com $\mathrm{X}$, ocorre quando $\sum b_{j}=0$ e $\sum c_{j}=0$, na primeira e na segunda equação.

Ademais, deve-se ressaltar que o modelo é sensível ao número de defasagens das variáveis que serão testadas, ocorrendo algum tipo de viés dependendo do número de defasagens ser muito baixo ou muito alto. Ao escolher um número de defasagens muito baixo, pode ocorrer de não estarem sendo consideradas no estudo algumas variáveis relevantes e que poderiam demonstrar causalidade onde defasagens menores não contenham informação suficiente para tal. Construir o modelo com um número de variáveis muito alto poderá resultar em variâncias com uma menor eficiência para o modelo, mas ainda assim os coeficientes das defasagens continuarão sendo calculados corretamente pelo método dos mínimos quadrados.

\subsection{Número de Defasagens}

De acordo com Maddala (1992), o número de defasagens escolhido pode ser considerado subjetivo, pois existe uma grande variedade de métodos para a escolha do número ideal de defasagens. Davidson e Mackinnon (1993) defendem que entre escolher um número pequeno 
de defasagens ou um número grande é preferível a segunda opção, pois escolhendo poucas variáveis pode-se incorrer no viés de excluir variáveis importantes e escolhendo um número maior incorre-se no viés de incluir variáveis irrelevantes, que é considerado um problema menor.

Considerando que há intenção de construir equações para prever os retornos futuros das ações utilizando os resultados do Teste de Causalidade de Granger e que durante a construção desses modelos as variáveis irrelevantes serão descartadas, decidiu-se que os testes serão realizados com até 6 meses de defasagem.

\subsection{Base de Dados}

As cotações das ações foram extraídas do site da Bolsa de Valores de São Paulo, após isso foram selecionadas as ações do setor de construção civil através do seguinte filtro:

1- Ativos que estejam na carteira do Índice Imobiliário (IMOB);

2- Ativos que estejam na carteira do Índice Bovespa (IBOV).

Garantindo desse modo uma liquidez mínima das ações escolhidas.

Sobre as empresas escolhidas a seguir é apresentada uma breve descrição:

A Brookfield é uma incorporadora nos segmentos comercial e residencial, desde empreendimentos econômicos aos imóveis de alto padrão. BRMalls Participações é uma holding que tem propriedade em cerca de cinquenta shopping centers no Brasil. A Cyrella Realty é uma construtora e incorporadora de imóveis residenciais operando em cerca de dezesseis estados brasileiros e mais de sessenta cidades. A Gafisa é uma construtora e incorporadora imobiliária com mais de cinquenta anos de tradição, fundada no Rio de Janeiro, dedicando-se à construção de imóveis residenciais e comerciais. A MRV sediada em Minas Gerais é uma construtora no segmento de casas e apartamentos, presente em cerca de cento e vinte cidades no Brasil. A
PDG Realty é uma construtora/incorporadora de imóveis com sede no Rio de Janeiro presente em dezenas localidades. A Rossi Residencial é uma construtora e incorporadora com sede em São Paulo com mais de cinquenta anos de tradição no mercado imobiliário. Atua em diversas regiões no Brasil.

Foram escolhidos indicadores econômicos para representar a inflação, índices financeiros e alguns índices de atividade. Como índices de preço, foram selecionados: o INCC, representando a inflação dos custos referentes à construção civil; o IPCA, que é o índice mais conhecido para medir a inflação brasileira; o IGP-M, utilizado principalmente para corrigir contratos de aluguel. O IPCA é calculado pelo Instituto Brasileiro de Geografia e Estatística (IBGE), enquanto a Fundação Getúlio Vargas (FGV) calcula o INCC e o IGP-M. Os dados dos três índices foram retirados do Portal Brasil (www.portalbrasil.net/indices).

Os índices financeiros escolhidos foram: a Taxa Selic, que será representada pelos Certificados de Depósito Interbancário (CDI), teve seus dados mensais também retirados do Portal Brasil; a taxa de câmbio, no formato (R\$/US\$), extraída do site do Banco Central (www.bcb.gov.br).

Por último, foram utilizados o PIB, Ibovespa e Taxa de Desocupação como índices de atividade e aquecimento da economia brasileira. O PIB teve seu banco de dados mensal retirado do IPEADATA 'www.ipeadata.gov.br). As cotações de fechamento do Índice Bovespa foram extraídas do site Bolsapt (www.bolsapt.com). A taxa de desocupação mensal foi obtida no banco de dados do IBGE (www.ibge.gov.br).

\subsection{Análise Descritiva dos Dados}

Após a coleta dos dados que foram utilizados no estudo, decidiu-se realizar a análise no período de 01 de agosto de 2007 até 31 de dezembro de 2011, pois observou-se que para ter todas 
as ações no mesmo período seria necessário atentar para o fato de que a primeira oferta publica da MRV foi em 23/07/2007. A tabela 1 mostra os códigos das ações escolhidas para compor o setor de construção civil, a data de sua primeira oferta, e seus pesos no Índice Imobiliário e também no Índice Bovespa.

Tabela 1: Informações das ações analisadas

\begin{tabular}{|cccccc|}
\hline Código & Ação & Tipo & $\mathbf{1}^{\circ}$ Oferta & Part. IMOB (\%) & Part. IBOV (\%) \\
\hline BISA3 & BROKFIELD & ON & $23 / 10 / 2006$ & $2,74 \%$ & $0,82 \%$ \\
\hline BRML3 & BR MALLS PAR & ON & $05 / 04 / 2007$ & $19,45 \%$ & $1,00 \%$ \\
\hline CYRE3 & CYRELA REALT & ON & $01 / 07 / 2005$ & $9,48 \%$ & $1,92 \%$ \\
\hline GFSA3 & GAFISA & ON & $17 / 02 / 2006$ & $4,35 \%$ & $1,81 \%$ \\
\hline MRVE3 & MRV & ON & $23 / 07 / 2007$ & $8,93 \%$ & $1,89 \%$ \\
\hline PDGR3 & PDGREALT & ON & $26 / 01 / 2007$ & $15,62 \%$ & $2,99 \%$ \\
\hline RSID3 & ROSSI RESID & ON & $05 / 01 / 2005$ & $3,45 \%$ & $1,35 \%$ \\
\hline TOTAL & & & & $64,02 \%$ & $11,78 \%$ \\
\hline
\end{tabular}

Fonte: www.bmfbovespa.com.br em 19/03/2012

A tabela 2 traz o resultado da análise descritiva das ações e dos indicadores selecionados.

Tabela 2: Estatísticas descritivas dos retornos mensais, período de ago/07 a dez/11.

\begin{tabular}{|c|c|c|c|c|c|c|}
\hline Variável & $\begin{array}{c}\text { Média } \\
\text { Aritmética } \\
\end{array}$ & Des vio Padrão & Mínimo & Máximo & Amplitude & Rent. Acumulada \\
\hline \multicolumn{7}{|l|}{ Ações } \\
\hline BISA3 & $-0,41 \%$ & $19,31 \%$ & $-32,31 \%$ & $92,31 \%$ & $124,62 \%$ & $-65,21 \%$ \\
\hline BRML3 & $1,62 \%$ & $12,28 \%$ & $-30,43 \%$ & $30,20 \%$ & $60,63 \%$ & $57,57 \%$ \\
\hline CYRE3 & $0,69 \%$ & $17,13 \%$ & $-45,64 \%$ & $48,26 \%$ & $93,90 \%$ & $-32,70 \%$ \\
\hline GFSA3 & $-0,77 \%$ & $18,52 \%$ & $-42,60 \%$ & $62,92 \%$ & $105,52 \%$ & $-72,52 \%$ \\
\hline MRVE3 & $1,52 \%$ & $16,98 \%$ & $-42,36 \%$ & $54,55 \%$ & $96,91 \%$ & $3,88 \%$ \\
\hline PDGR3 & $1,24 \%$ & $15,35 \%$ & $-31,48 \%$ & $59,20 \%$ & $90,68 \%$ & $8,76 \%$ \\
\hline RSID3 & $-0,10 \%$ & $21,31 \%$ & $-46,00 \%$ & $104,05 \%$ & $150,05 \%$ & $-66,53 \%$ \\
\hline \multicolumn{7}{|c|}{ Indicadores Financeiros } \\
\hline CDI & $0,87 \%$ & $0,13 \%$ & $0,59 \%$ & $1,17 \%$ & $0,58 \%$ & $57,99 \%$ \\
\hline Dolar & $0,13 \%$ & $5,31 \%$ & $-9,43 \%$ & $17,13 \%$ & $26,56 \%$ & $-0,09 \%$ \\
\hline \multicolumn{7}{|c|}{ Indicadores de Preços } \\
\hline INCC & $0,59 \%$ & $0,59 \%$ & $-0,25 \%$ & $2,94 \%$ & $3,19 \%$ & $36,79 \%$ \\
\hline IPCA & $0,46 \%$ & $0,22 \%$ & $0,00 \%$ & $0,83 \%$ & $0,83 \%$ & $27,20 \%$ \\
\hline IGPM & $0,55 \%$ & $0,63 \%$ & $-0,74 \%$ & $1,98 \%$ & $2,72 \%$ & $33,72 \%$ \\
\hline \multicolumn{7}{|c|}{ Indicadores de Atividade } \\
\hline IBOV & $0,36 \%$ & $7,30 \%$ & $-24,80 \%$ & $15,55 \%$ & $40,35 \%$ & $4,75 \%$ \\
\hline PIB & $0,98 \%$ & $3,68 \%$ & $-9,66 \%$ & $6,99 \%$ & $16,65 \%$ & $61,60 \%$ \\
\hline Txdes & $-1,15 \%$ & $5,99 \%$ & $-10,53 \%$ & $20,59 \%$ & $31,12 \%$ & $-50,53 \%$ \\
\hline
\end{tabular}

\section{Fonte: Elaborado pelos autores}

Analisando a tabela 2, nota-se que o CDI foi o investimento com a maior rentabilidade no período, sendo a ação da BRML3 a única que se aproximou do CDI. O PIB do período teve um crescimento médio de $0,98 \%$, enquanto a taxa de desemprego diminuía com mais rapidez, em torno $-1,15 \%$ ao mês. O destaque negativo ficou com a GFSA3, com a menor média de rentabilidade mensal e também acumulada. Abaixo está a tabela 3, que apresenta o Coeficiente de 
Correlação de Pearson para todas as variáveis do estudo, com $\mathrm{o}$ teste $\mathrm{t}$ associado à hipótese nula dos coeficientes serem iguais a zero.

Tabela 3: Coeficiente de Correlação de Pearson das variáveis, período de ago/07 a dez/11.

\begin{tabular}{|c|c|c|c|c|c|c|c|c|}
\hline Variável & CDI & Dolar & INCC & IPCA & IGPM & IBOV & PIB & Txdes \\
\hline \multicolumn{9}{|l|}{ Ações } \\
\hline \multirow{2}{*}{ BISA3 } & $(0,380)$ & $(0,568)$ & $(0,193)$ & $(0,093)$ & $(0,213)$ & 0,641 & $(0,001)$ & $(0,105)$ \\
\hline & $0,005 * * *$ & $0,000 * * *$ & 0,166 & 0,507 & 0,126 & $0,000 * * *$ & 0,994 & 0,455 \\
\hline \multirow{2}{*}{ BRML3 } & $(0,227)$ & $(0,283)$ & $(0,160)$ & $(0,070)$ & $(0,255)$ & 0,604 & 0,011 & 0,102 \\
\hline & 0,103 & $0,04 * *$ & 0,253 & 0,616 & $0,066^{*}$ & $0,000 * * *$ & 0,936 & 0,469 \\
\hline \multirow{2}{*}{ CYRE3 } & $(0,224)$ & $(0,616)$ & $(0,184)$ & $(0,304)$ & $(0,325)$ & 0,770 & 0,088 & $(0,072)$ \\
\hline & 0,107 & $0,000 * * *$ & 0,186 & $0,027 * *$ & $0,018 * *$ & $0,000 * * *$ & 0,531 & 0,607 \\
\hline \multirow{2}{*}{ GFSA 3} & $(0,219)$ & $(0,550)$ & $(0,262)$ & $(0,222)$ & $(0,267)$ & 0,684 & $(0,008)$ & 0,002 \\
\hline & 0,115 & $0,000 * * *$ & $0,058^{*}$ & 0,110 & $0,053^{*}$ & $0,000 * * *$ & 0,954 & 0,986 \\
\hline \multirow{2}{*}{ MRVE3 } & $(0,279)$ & $(0,697)$ & $(0,143)$ & $(0,128)$ & $(0,260)$ & 0,765 & 0,229 & $(0,006)$ \\
\hline & $0,043 * *$ & $0,000 * * *$ & 0,306 & 0,363 & $0,06 *$ & $0,000 * * *$ & $0,1^{*}$ & 0,966 \\
\hline \multirow{2}{*}{ PDGR3 } & $(0,191)$ & $(0,644)$ & $(0,187)$ & $(0,104)$ & $(0,210)$ & 0,736 & 0,188 & 0,002 \\
\hline & 0,171 & $0,000 * * *$ & 0,181 & 0,457 & 0,130 & $0,000 * * *$ & 0,177 & 0,986 \\
\hline \multirow{2}{*}{ RSID3 } & $(0,240)$ & $(0,614)$ & $(0,231)$ & $(0,137)$ & $(0,240)$ & 0,681 & 0,146 & 0,014 \\
\hline & $0,084 *$ & $0,000 * * *$ & $0,096 *$ & 0,329 & $0,083 *$ & $0,000 * * *$ & 0,297 & 0,922 \\
\hline \multicolumn{9}{|c|}{ Indicadores Financeiros } \\
\hline \multirow{2}{*}{ CDI } & 1,000 & 0,362 & 0,143 & $(0,119)$ & $(0,029)$ & $(0,386)$ & $(0,002)$ & 0,077 \\
\hline & $0,000 * * *$ & $0,008 * * *$ & 0,306 & 0,398 & 0,834 & $0,004 * * *$ & 0,987 & 0,585 \\
\hline \multirow{2}{*}{ Dolar } & 0,362 & 1,000 & 0,017 & 0,005 & $(0,025)$ & $(0,675)$ & $(0,217)$ & 0,049 \\
\hline & $0,008 * * *$ & $0,000 * * *$ & 0,906 & 0,970 & 0,857 & $0,000 * * *$ & 0,119 & 0,728 \\
\hline \multicolumn{9}{|c|}{ Indicadores de Preços } \\
\hline \multirow{2}{*}{ INCC } & 0,143 & 0,017 & 1,000 & 0,208 & 0,377 & $(0,260)$ & 0,281 & $(0,050)$ \\
\hline & 0,306 & 0,906 & $0,000 * * *$ & 0,134 & $0,005 * * *$ & $0,061 *$ & $0,041 * *$ & 0,724 \\
\hline \multirow{2}{*}{ IPCA } & $(0,119)$ & 0,005 & 0,208 & 1,000 & 0,513 & $(0,117)$ & $(0,083)$ & 0,209 \\
\hline & 0,398 & 0,970 & 0,134 & $0,000 * * *$ & $0,000 * * *$ & 0,405 & 0,554 & 0,133 \\
\hline \multirow{2}{*}{ IGPM } & $(0,029)$ & $(0,025)$ & 0,377 & 0,513 & 1,000 & $(0,232)$ & 0,063 & $(0,065)$ \\
\hline & 0,834 & 0,857 & $0,005 * * *$ & $0,000 * * *$ & $0,000 * * *$ & $0,094 *$ & 0,655 & 0,641 \\
\hline \multicolumn{9}{|c|}{ Indicadores de Atividade } \\
\hline \multirow{2}{*}{ IBOV } & $(0,386)$ & $(0,675)$ & $(0,260)$ & $(0,117)$ & $(0,232)$ & 1,000 & 0,093 & $(0,044)$ \\
\hline & $0,004 * * *$ & $0,000 * * *$ & $0,061 *$ & 0,405 & $0,094 *$ & $0,000 * * *$ & 0,509 & 0,755 \\
\hline \multirow{2}{*}{$\mathrm{PIB}$} & $(0,002)$ & $(0,217)$ & 0,281 & $(0,083)$ & 0,063 & 0,093 & 1,000 & $(0,149)$ \\
\hline & 0,987 & 0,119 & $0,041 * *$ & 0,554 & 0,655 & 0,509 & $0,000 * * *$ & 0,288 \\
\hline \multirow{2}{*}{ Txdes } & 0,077 & 0,049 & $(0,050)$ & 0,209 & $(0,065)$ & $(0,044)$ & $(0,149)$ & 1,000 \\
\hline & 0,585 & 0,728 & 0,724 & 0,133 & 0,641 & 0,755 & 0,288 & $0,000 * * *$ \\
\hline
\end{tabular}

Fonte: Elaborado pelos autores

Interessante notar que na relação entre as variáveis dependentes e independentes o IBOV e o Dólar são as variáveis com as maiores correlações, tendo seu valor máximo de 0,77 , entre

terceiro lugar, rejeitando a hipótese nula para duas ações ao grau de
CYRE3 e IBOV. Essas duas variáveis explicativas são também as únicas que rejeitam a hipótese nula para todas as ações, com significância de pelo menos $5 \%$. As correlações com o CDI vêm em $5 \%$. Os indicadores de preço mostraram ter correlações baixas, e considerando 
uma significância de $10 \%$ é possível dizer que o INCC se mostra diferente de zero apenas para GFSA3, o IPCA pode ser considerado diferente de zero apenas para CYRE3, e o IGP-M seria o índice mais influente destes três, considerado significativo para BRML3, CYRE3, GFSA3, MRVE3 e RSID3. O PIB e a Taxa de Desemprego mostraram ter correlações bem baixas para todas as ações e com significância estatística somente entre o PIB e MRVE3 para o nível de $10 \%$.

Ao analisar somente as correlações entre as variáveis independentes, notam-se as maiores correlações entre o IBOV e o Dólar, no valor de -0,675. Em seguida, vêm a relação negativa do CDI com o IBOV de $-0,386$ e positiva com o Dólar de 0,362 , ambas com significância de $1 \%$. Dentre as outras variáveis, somente o IGP-M demonstrou ter uma correlação maior com o IPCA, de 0,513, o que pode ser explicado pelo fato do IPCA representar 60\% da composição do IGP-M3.

Tabela 4: Teste de Dickey-Fuller Aumentado, período de ago/07 a dez/11

\begin{tabular}{|c|c|c|c|c|c|c|}
\hline \multirow[t]{2}{*}{ Variável } & \multicolumn{2}{|c|}{ S/ Intercepto e S/ Tendên cia } & \multicolumn{2}{|c|}{$\mathrm{C} /$ Intercepto e S/ Tendência } & \multicolumn{2}{|c|}{$\mathrm{C} /$ Inter cepto e $\mathrm{C} /$ Tendên cia } \\
\hline & $\tau$ & p-val or & $\tau \mu$ & p-val or & $\tau \tau$ & p-valor \\
\hline \multicolumn{7}{|l|}{ Ações } \\
\hline BISA3 & $-6,20$ & $<0,0001$ & $-6,14$ & 0,0001 & $-6,06$ & $<0,0001$ \\
\hline BRML3 & $-8,25$ & $<0,0001$ & $-8,36$ & 0,0001 & $-8,41$ & $<0,0001$ \\
\hline CYRE3 & $-6,67$ & $<0,0001$ & $-6,62$ & 0,0001 & $-6,59$ & $<0,0001$ \\
\hline GFSA 3 & $-7,36$ & $<0,0001$ & $-7,29$ & 0,0001 & $-7,42$ & $<0,0001$ \\
\hline MRVE3 & $-5,53$ & $<0,0001$ & $-5,50$ & 0,0001 & $-5,45$ & 0,0002 \\
\hline PDGR3 & $-6,61$ & $<0,0001$ & $-6,56$ & 0,0001 & $-6,50$ & $<0,0001$ \\
\hline RSID3 & $-6,51$ & $<0,0001$ & $-6,45$ & 0,0001 & $-6,38$ & $<0,0001$ \\
\hline \multicolumn{7}{|c|}{ Indicadores Financeir os } \\
\hline CDI (1) & $-11,20$ & $<0,0001$ & $-11,09$ & 0,0001 & $-11,04$ & $<0,0001$ \\
\hline Dolar & $-6,09$ & $<0,0001$ & $-6,03$ & 0,0001 & $-6,00$ & $<0,0001$ \\
\hline \multicolumn{7}{|c|}{ Indicadores de Preços } \\
\hline INCC & $-2,80$ & 0,0060 & $-4,30$ & 0,0012 & $-4,31$ & 0,0064 \\
\hline IPCA (1) & $-7,03$ & $<0,0001$ & $-6,96$ & 0,0001 & $-6,88$ & $<0,0001$ \\
\hline IGPM & $-2,73$ & 0,0072 & $-3,56$ & 0,0099 & $-3,61$ & 0,0390 \\
\hline \multicolumn{7}{|c|}{ Indicadores de Atividade } \\
\hline IBOV & $-5,67$ & $<0,0001$ & $-5,63$ & 0,0001 & $-5,59$ & 0,0002 \\
\hline PIB & $-6,78$ & $<0,0001$ & $-7,13$ & 0,0001 & $-7,06$ & $<0,0001$ \\
\hline Txdes & $-6,53$ & $<0,0001$ & $-6,71$ & 0,0001 & $-6,69$ & $<0,0001$ \\
\hline
\end{tabular}

(1) Série diferen ciada de ordem 1

\section{ANÁLISE DOS RESULTADOS}

O teste de raiz unitária de DickeyFuller Aumentado foi realizado em todas as variáveis utilizando o software estatístico SAS. Todas as séries, com exceção do CDI e do IPCA, foram consideradas estacionárias usando os três modelos apresentados pelo teste ADF, $1 \%$, dependendo da série. O CDI e o IPCA só foram considerados estacionários após a aplicação de diferenciação. Com a estacionariedade das séries, fica confirmada uma das premissas necessárias para o Teste de Causalidade de Granger. com significância estatística de $5 \%$ ou 


\subsection{Resultados do Teste de Causalidade de Granger}

Os testes de causalidade foram realizados fazendo uso do software SPSS 15.0 e do Microsoft Excel. Esses testes têm como objetivo medir se uma variável dependente é influenciada por valores passados de uma variável independente, na presença de valores defasados da variável dependente, dizendo-se então que a variável condicional causa a variável condicionada. Neste estudo, foram feitos testes de Causalidade de Granger para as sete ações selecionadas em relação aos oito indicadores escolhidos. Os testes foram bivariados, o que significa utilizar as equações definidas na seção 3.2 para identificar possíveis causalidades entre as variáveis.

Decidiu-se realizar o teste utilizando cada ação individualmente, ao invés de construir um índice com todas as ações, para que se pudesse capturar a influência dos indicadores em cada ativo de modo individualizado. Desse modo, é possível distinguir se alguma ação tem comportamento diferente das outras ações do grupo, algo que seria impossível distinguir ao utilizar apenas um índice setorial. Outro parâmetro do estudo envolve a escolha do número de defasagens, que em todos os casos foi considerada de seis meses. Testou-se a hipótese nula de que "não há causalidade" e foi considerado um nível de significância de $10 \%$. Como o objetivo do estudo é relativo a medir os retornos das ações com os indicadores macroeconômicos, não foram realizados testes de causalidade entre as ações e nem entre os indicadores econômicos. Segue tabela 5 com os resultados encontrados: 
Tabela 5: Teste de Causalidade de Granger

\begin{tabular}{|c|c|c|c|c|c|}
\hline \multicolumn{3}{|c|}{ Indicadores -> Ações } & \multicolumn{3}{|c|}{ Ações -> Indicadores } \\
\hline Hipótese Nula & Estatística F & $p$-valor & Hipótese Nula & Estatística F & $\mathrm{p}$-valor \\
\hline CDI não causa BISA3 & 1,909 & 0,108 & BISA3 não causa CDI & 0,781 & 0,590 \\
\hline Dolar não causa BISA3 & 1,129 & 0,367 & BISA3 não causa Dolar & 0,998 & 0,443 \\
\hline INCC não causa BISA3 & 2,167 & $0,071^{*}$ & BISA3 não causa INCC & 0,837 & 0,550 \\
\hline IPCA não causa BISA3 & 1,483 & 0,213 & BISA3 não causa IPCA & 0,333 & 0,915 \\
\hline IGPM não causa BISA3 & 1,599 & 0,178 & BISA3 não causa IGPM & 0,448 & 0,841 \\
\hline IBOV não causa BISA3 & 2,097 & $0,079^{*}$ & BISA3 não causa IBOV & 0,620 & 0,713 \\
\hline PIB não causa BISA3 & 0,956 & 0,469 & BISA3 não causa PIB & 1,972 & $0,097^{*}$ \\
\hline TxDes não causa BISA3 & 2,207 & $0,066^{*}$ & BISA3 não causa TxDes & 0,602 & 0,727 \\
\hline CDI não causa BRML3 & 1,838 & 0,121 & BRML3 não causa CDI & 0,158 & 0,986 \\
\hline Dolar não causa BRML3 & 1,189 & 0,336 & BRML3 não causa Dolar & 0,876 & 0,522 \\
\hline INCC não causa BRML3 & 1,591 & 0,180 & BRML3 não causa INCC & 1,020 & 0,429 \\
\hline IPCA não causa BRML3 & 0,569 & 0,752 & BRML3 não causa IPCA & 1,517 & 0,202 \\
\hline IGPM não causa BRML3 & 1,051 & 0,410 & BRML3 não causa IGPM & 1,348 & 0,264 \\
\hline IBOV não causa BRML3 & 1,665 & 0,160 & BRML3 não causa IBOV & 0,235 & 0,962 \\
\hline PIB não causa BRML3 & 0,966 & 0,463 & BRML3 não causa PIB & 0,315 & 0,925 \\
\hline TxDes não causa BRML3 & 1,244 & 0,309 & BRML3 não causa TxDes & 0,895 & 0,510 \\
\hline CDI não causa CYRE3 & 2,191 & $0,068^{*}$ & CYRE3 não causa CDI & 0,383 & 0,885 \\
\hline Dolar não causa CYRE3 & 1,353 & 0,262 & CYRE3 não causa Dolar & 0,419 & 0,861 \\
\hline INCC não causa CYRE3 & 1,634 & 0,168 & CYRE3 não causa INCC & 0,759 & 0,607 \\
\hline IPCA não causa CYRE3 & 1,240 & 0,311 & CYRE3 não causa IPCA & 0,523 & 0,787 \\
\hline IGPM não causa CYRE3 & 1,597 & 0,178 & CYRE3 não causa IGPM & 0,733 & 0,626 \\
\hline IBOV não causa CYRE3 & 2,486 & $0,042^{* *}$ & CYRE3 não causa IBOV & 0,381 & 0,886 \\
\hline PIB não causa CYRE3 & 2,000 & $0,093^{*}$ & CYRE3 não causa PIB & 1,521 & 0,201 \\
\hline TxDes não causa CYRE3 & 2,866 & $0,023^{* \star}$ & CYRE3 não causa TxDes & 0,665 & 0,678 \\
\hline CDI não causa GFSA3 & 1,461 & 0,221 & GFSA3 não causa CDI & 1,290 & 0,288 \\
\hline Dolar não causa GFSA3 & 1,587 & 0,181 & GFSA3 não causa Dolar & 1,626 & 0,170 \\
\hline INCC não causa GFSA3 & 2,092 & $0,08^{\star}$ & GFSA3 não causa INCC & 0,642 & 0,696 \\
\hline IPCA não causa GFSA3 & 0,642 & 0,696 & GFSA3 não causa IPCA & 0,799 & 0,577 \\
\hline IGPM não causa GFSA3 & 1,642 & 0,166 & GFSA3 não causa IGPM & 0,838 & 0,549 \\
\hline IBOV não causa GFSA3 & 1,732 & 0,144 & GFSA3 não causa IBOV & 1,477 & 0,216 \\
\hline PIB não causa GFSA3 & 1,028 & 0,424 & GFSA3 não causa PIB & 1,837 & 0,121 \\
\hline TxDes não causa GFSA3 & 3,834 & $0,005^{* \star *}$ & GFSA3 não causa TxDes & 0,647 & 0,692 \\
\hline CDI não causa MRVE3 & 1,749 & 0,140 & MRVE3 não causa CDI & 0,302 & 0,932 \\
\hline Dolar não causa MRVE3 & 1,048 & 0,413 & MRVE3 não causa Dolar & 0,692 & 0,658 \\
\hline INCC não causa MRVE3 & 3,192 & $0,014^{* *}$ & MRVE3 não causa INCC & 0,705 & 0,648 \\
\hline IPCA não causa MRVE3 & 0,978 & 0,455 & MRVE3 não causa IPCA & 0,197 & 0,975 \\
\hline IGPM não causa MRVE3 & 2,401 & $0,048^{* *}$ & MRVE3 não causa IGPM & 0,617 & 0,715 \\
\hline IBOV não causa MRVE3 & 2,660 & $0,032^{* *}$ & MRVE3 não causa IBOV & 0,603 & 0,726 \\
\hline PIB não causa MRVE3 & 1,696 & 0,152 & MRVE3 não causa PIB & 1,319 & 0,276 \\
\hline TxDes não causa MRVE3 & 0,795 & 0,580 & MRVE3 não causa TxDes & 0,516 & 0,792 \\
\hline CDI não causa PDGR3 & 1,809 & 0,127 & PDGR3 não causa CDI & 0,847 & 0,543 \\
\hline Dolar não causa PDGR3 & 1,176 & 0,342 & PDGR3 não causa Dolar & 1,365 & 0,257 \\
\hline INCC não causa PDGR3 & 2,183 & $0,069^{*}$ & PDGR3 não causa INCC & 1,004 & 0,439 \\
\hline IPCA não causa PDGR3 & 1,463 & 0,220 & PDGR3 não causa IPCA & 0,453 & 0,838 \\
\hline IGPM não causa PDGR3 & 2,123 & $0,076^{*}$ & PDGR3 não causa IGPM & 0,514 & 0,794 \\
\hline IBOV não causa PDGR3 & 2,105 & $0,078^{*}$ & PDGR3 não causa IBOV & 0,760 & 0,606 \\
\hline PIB não causa PDGR3 & 2,079 & $0,082^{*}$ & PDGR3 não causa PIB & 2,095 & $0,08^{*}$ \\
\hline TxDes não causa PDGR3 & 1,889 & 0,111 & PDGR3 não causa TxDes & 0,526 & 0,785 \\
\hline CDI não causa RSID3 & 1,767 & 0,136 & RSID3 não causa CDI & 0,573 & 0,749 \\
\hline Dolar não causa RSID3 & 1,292 & 0,287 & RSID3 não causa Dolar & 0,635 & 0,701 \\
\hline IINCC não causa RSID3 & 1,842 & 0,120 & RSID3 não causa INCC & 1,444 & 0,227 \\
\hline IPCA não causa RSID3 & 0,849 & 0,542 & RSID3 não causa IPCA & 0,315 & 0,925 \\
\hline IGPM não causa RSID3 & 1,932 & 0,104 & RSID3 não causa IGPM & 0,351 & 0,905 \\
\hline IBOV não causa RSID3 & 2,216 & $0,065^{*}$ & RSID3 não causa IBOV & 0,832 & 0,553 \\
\hline PIB não causa RSID3 & 2,594 & $0,035^{* \star}$ & RSID3 não causa PIB & 1,182 & 0,339 \\
\hline TxDes não causa RSID3 & 2,825 & $0,024^{* \star}$ & RSID3 não causa TxDes & 0,413 & 0,865 \\
\hline
\end{tabular}

Fonte: Elaborado pelos autores 
Os resultados mostram que a única ação que não causa e nem é causada por nenhum indicador é a BRML3, com todos os seus testes de significância acima de $12 \%$, o que demonstra independência desta ação em relação aos indicadores. Todas as outras ações são causadas, no sentido de Granger, por pelo menos 2 indicadores econômicos. O IBOV se mostra mais uma vez como o indicador mais importante, causando cinco das sete ações. O INCC, PIB e a Taxa de Desemprego aparecem causando 3, 3 e 4 ações, respectivamente. Do lado direito da tabela 5, verifica-se que o único indicador causado pelas ações é o PIB, o que significa que os retornos de BISA3 e PDGR3 acabam de algum modo precedendo os retornos desse indicador da atividade econômica. Das causalidades encontradas, a ação PDGR3 é a única com causalidade bilateral ou retroalimentação, causando e sendo causada pelo PIB. Os outros casos se referem apenas a causalidades unilaterais.

Visto que somente uma ação não é precedida por mudanças nos retornos dos indicadores macroeconômicos, a conclusão é de que as ações são causadas, no sentido de Granger, pelos indicadores macroeconômicos. No entanto, essa "causalidade" não é garantia de que seja possível construir um bom modelo relacionando os retornos acionários aos retornos passados dos indicadores. Segue tabela 6 com o resultado da análise de regressão multivariada dos retornos das ações em relações aos retornos passados dos indicadores que demonstraram ter poder causal, ressaltando que foram usados 6 meses de defasagens dos indicadores e utilizou-se a letra "D" associada a um número para representar o grau de defasagem do indicador:

Tabela 6: Modelos de regressão multivariados precedentes

\begin{tabular}{|c|c|c|c|c|c|c|c|c|c|c|}
\hline \multirow[t]{2}{*}{ Ações } & \multicolumn{9}{|c|}{ Variáveis Dependentes } & \multirow[t]{2}{*}{$\mathbf{R}^{2}$ Ajustado } \\
\hline & Constante & INCC D1 & INCC D4 & IBOV D1 & IBOV D3 & IBOV D4 & IBOV D6 & Txdes D3 & Txdes D5 & \\
\hline \multirow{2}{*}{ BISA3 } & 0,190 & $-17,798$ & $-8,948$ & $-0,547$ & 0,710 & 0,485 & $-0,662$ & 1,369 & 0,955 & \multirow{2}{*}{0,632} \\
\hline & $0,000 * * *$ & $0,000 * * *$ & $0,014 * *$ & $0,060^{*}$ & $0,017 * *$ & $0,064 *$ & $0,016^{* * *}$ & $0,000 * * *$ & $0,016^{* *}$ & \\
\hline & - & - & - & - & - & - & - & - & - & \\
\hline \multirow{2}{*}{ BRML3 } & - & - & - & - & - & - & - & - & - & \multirow{2}{*}{ - } \\
\hline & - & - & - & - & - & - & - & - & - & \\
\hline & Constante & CDID4 & CDID5 & CDID6 & IBOV D6 & PIB D6 & Txdes D2 & Txdes D6 & - & \\
\hline \multirow{2}{*}{ CYRE3 } & 0,015 & $-66,655$ & $-90,620$ & $-81,638$ & $-1,409$ & 1,148 & 0,847 & 0,824 & - & \multirow{2}{*}{0,517} \\
\hline & 0,428 & $0,012 * *$ & $0,001 * * *$ & $0,004 * * *$ & $0,000 * * *$ & $0,047 * *$ & $0,007 * * *$ & $0,016 * *$ & - & \\
\hline & Constante & INCC D1 & INCC D4 & Txdes D2 & Txdes D3 & Txdes D5 & Txdes D6 & - & - & \\
\hline \multirow{2}{*}{ GFSA3 } & 0,135 & $-10,403$ & $-8,892$ & 0,764 & 0,897 & 0,744 & 1,189 & - & - & \multirow{2}{*}{0,367} \\
\hline & $0,003 * * *$ & $0,021 * *$ & $0,020^{* *}$ & $0,049 * *$ & $0,021 * *$ & 0,094* & $0,003 * * *$ & - & - & \\
\hline & Constante & INCC D4 & IGPMD3 & IBOV D6 & - & - & - & - & - & \\
\hline \multirow{2}{*}{ MRVE3 } & 0,131 & $-9,839$ & $-9,186$ & $-0,982$ & - & - & - & - & - & \multirow{2}{*}{0,466} \\
\hline & $0,000 * * *$ & $0,003 * * *$ & $0,004 * * *$ & $0,000 * * *$ & - & - & - & - & - & \\
\hline & \begin{tabular}{|l|} 
Constante \\
\end{tabular} & INCC D1 & INCC D4 & IGPM D3 & IGPM D5 & IBOV D6 & - & - & - & \\
\hline \multirow{2}{*}{ PDGR3 } & 0,121 & $-6,834$ & $-8,521$ & $-7,266$ & 5,565 & $-0,728$ & - & - & - & \multirow{2}{*}{0,380} \\
\hline & $0,001 * * *$ & $0,041 * *$ & $0,013 * *$ & $0,028 * *$ & $0,089 *$ & $0,007 * * *$ & - & - & - & \\
\hline & Constante & IBOV D3 & IBOV D6 & PIB D2 & PIB D4 & TxDes D6 & - & - & - & \\
\hline \multirow{2}{*}{ RSID3 } & 0,065 & 0,742 & $-1,111$ & $-1,675$ & $-2,492$ & 1,588 & - & - & - & \multirow{2}{*}{0,369} \\
\hline & $0,034 * *$ & $0,049 * *$ & $0,004 * * *$ & $0,038 * *$ & $0,003 * * *$ & $0,003 * * *$ & - & - & - & \\
\hline
\end{tabular}


A análise de regressão multivariada dos retornos das ações com os indicadores macroeconômicos foi feita no software SPSS 15.0. Foi utilizado o método de construção de equações backward, que significa realizar a regressão com todos os indicadores e remover aqueles que tiverem uma significância acima de $10 \%$.

Percebe-se que estes modelos precedentes são complexos, possuindo uma quantidade grande de variáveis e um $R^{2}$ ajustado em entre 0,367 e 0,632 . Analisando a equação da BISA3, é difícil entender a forma como está ocorrendo o ajuste do modelo, pois existem muitas variáveis dependentes e o IBOV, por exemplo, possui coeficientes positivos e negativos dependendo da defasagem, de modo que não é simples conceituar se sua influência é positiva ou negativa.

\section{Reais}

\subsection{Modelo versus Retornos}

Após a construção dos modelos apresentados na tabela 6 , pensa-se que seria interessante comparar os resultados calculados por essas equações com retornos reais, pois esta seria uma forma prática de saber se os modelos se mostram adequados a dados futuros. Construiu-se então a tabela 7, que compara os retornos dos modelos, chamados de Modelo P, com os retornos reais do mercado acionário, chamados de Real, durante um período de 6 meses, de janeiro a junho de 2012.

Embora um semestre possa ser considerado um período estatisticamente curto, a comparação dos retornos mostra que os modelos parecem se afastar muito dos retornos reais encontrados para as 6 ações. A equação da ação BISA3, por exemplo, possui nove variáveis dependentes, mas a impressão que fica é de que essas variáveis serviram apenas para explicar o período em que foi construído o modelo, e não conseguem ajudar a estimar retornos que se aproximem dos reais, embora o $R^{2}$ ajustado seja de 0,632.

\section{CONSIDERAÇÕES FINAIS}

Este estudo teve como objetivo descobrir se há alguma relação causal entre os retornos acionários das empresas da construção civil brasileira e os indicadores macroeconômicos brasileiros. Optou-se por usar o Teste de Causalidade de Granger para descobrir a existência ou não da causalidade.

A primeira etapa do trabalho foi utilizar o teste de raiz unitária de DickeyFuller Aumentado em todas as séries para detectar se estas eram estacionárias e realizar o devido tratamento naquelas que não fossem. Em seguida, foi aplicado o Teste de Causalidade de Granger nas variáveis, o qual mostrou que alguns indicadores exerciam uma influência atrasada nas ações, enquanto somente duas ações causavam, no sentido de Granger, um indicador, que foi o PIB. Conforme tabela 5 , os indicadores que causavam as ações foram: Certificado de Depósitos Interbancários, Índice Bovespa, Taxa de Desemprego, Produto Interno Bruto, Índice Nacional de Custo Civil e o Índice Geral de Preços do Mercado. O maior impacto sob as ações é feito pelo Ibovespa, mostrando o poder que este índice tem nas ações do setor imobiliário. Houve a tentativa de se criar modelos de regressão utilizando as variáveis defasadas, mas estes modelos se mostraram complexos, e com resultados um pouco distantes dos retornos reais das ações em um período futuro selecionado para teste.

Sendo assim, observou-se que existe uma relação de causalidade entre o retorno das ações do setor de construção civil brasileiro e alguns dos indicadores macroeconômicos selecionados por este estudo. Mostrando que a maioria das ações do setor, 6 entre 7, sofre um efeito atrasado das variações que ocorrem nos indicadores. Concluindo então que as mudanças nas variáveis econômicas precedem as do setor imobiliário. 
Tabela 7: Comparação entre os modelos e dados reais, período de jan/12 a jun/12.

\begin{tabular}{|c|c|c|c|c|c|}
\hline \multicolumn{3}{|c|}{ BISA3 } & \multicolumn{3}{|c|}{ BRML3 } \\
\hline Mês & Modelo P & Real & Mês & Modelo P & Real \\
\hline janeiro-12 & $19,75 \%$ & $24,04 \%$ & janeiro-12 & - & $5,24 \%$ \\
\hline fevereiro-12 & $-12,70 \%$ & $3,17 \%$ & fevereiro-12 & - & $13,10 \%$ \\
\hline março-12 & $-7,96 \%$ & $-11,80 \%$ & março-12 & - & $5,17 \%$ \\
\hline abril-12 & $23,66 \%$ & $-15,20 \%$ & abril-12 & - & $3,14 \%$ \\
\hline maio-12 & $5,94 \%$ & $-30,66 \%$ & maio-12 & - & $-8,19 \%$ \\
\hline junho-12 & $18,48 \%$ & $-7,28 \%$ & junho-12 & - & $6,48 \%$ \\
\hline \multicolumn{3}{|c|}{ CYRE3 } & & GFSA3 & \\
\hline Mês & Modelo P & Real & Mês & Modelo P & Real \\
\hline janeiro-12 & $-7,19 \%$ & $9,03 \%$ & janeiro-12 & $-3,63 \%$ & $15,78 \%$ \\
\hline fevereiro-12 & $8,76 \%$ & $3,03 \%$ & fevereiro-12 & $-14,44 \%$ & $6,46 \%$ \\
\hline marco-12 & $40,35 \%$ & $-11,31 \%$ & marco-12 & $5,87 \%$ & $-11,52 \%$ \\
\hline abril-12 & $-5,86 \%$ & $-8,22 \%$ & abril-12 & $13,60 \%$ & $-20,63 \%$ \\
\hline maio-12 & $4,63 \%$ & $-3,57 \%$ & maio-12 & $-11,71 \%$ & $-29,16 \%$ \\
\hline junho-12 & $-2,75 \%$ & $2,71 \%$ & junho-12 & $-2,10 \%$ & $3,95 \%$ \\
\hline \multicolumn{3}{|c|}{ MRVE3 } & \multicolumn{3}{|c|}{ PDGR3 } \\
\hline Mês & Modelo P & Real & Mês & Modelo P & Real \\
\hline janeiro-12 & $12,53 \%$ & $25,70 \%$ & janeiro-12 & $12,93 \%$ & $20,00 \%$ \\
\hline fevereiro-12 & $10,17 \%$ & $-1,98 \%$ & fevereiro-12 & $6,93 \%$ & $0,55 \%$ \\
\hline março-12 & $14,40 \%$ & $-8,35 \%$ & março-12 & $13,11 \%$ & $-16,42 \%$ \\
\hline abril-12 & $-1,53 \%$ & $-15,92 \%$ & abril-12 & $0,29 \%$ & $-30,02 \%$ \\
\hline maio-12 & $7,39 \%$ & $-25,67 \%$ & maio-12 & $0,99 \%$ & $-28,66 \%$ \\
\hline junho-12 & $6,44 \%$ & $5,68 \%$ & junho-12 & $-4,88 \%$ & $3,24 \%$ \\
\hline \multicolumn{3}{|c|}{ RSID3 } & & & \\
\hline Mês & Modelo P & Real & & & \\
\hline janeiro-12 & $21,37 \%$ & $18,00 \%$ & & & \\
\hline fevereiro-12 & $0,72 \%$ & $4,15 \%$ & & & \\
\hline março-12 & $20,46 \%$ & $-4,46 \%$ & & & \\
\hline abril-12 & $0,31 \%$ & $-21,57 \%$ & & & \\
\hline maio-12 & $0,84 \%$ & $-34,08 \%$ & & & \\
\hline junho-12 & $-9,30 \%$ & $-9,04 \%$ & & & \\
\hline
\end{tabular}

Fonte: Elaborado pelos autores

Fica como sugestão que trabalhos futuros possam realizar outros tipos de teste de causalidade no setor estudado com intuito de saber se os resultados encontrados seriam similares. Seria interessante também realizar testes causais entre as ações e seus indicadores fundamentalistas, bem como outras variáveis externas não contempladas neste trabalho, mas que possam influenciar o setor de construção, como a disponibilidade de crédito na economia brasileira e a inadimplência. Sugere-se ainda usar os estudos causais em outros grupos de ações do mercado brasileiro para saber como estes seriam afetados pelos indicadores aqui utilizados e comparar com o setor de construção civil.

\section{REFERÊNCIAS}

ADARAMOLA, Anthony O. Oil Price Shocks and Stock Market Behaviour: The Nigerian Experience. Journal Economics, vol. 3, n. 1, 2012, p 19-24.

ADRANGI, Bahram; CHATRATH, Arjun; SANVICENTE, Antonio Z. Inflation, Output, And Stock Prices: Evidence From Brazil. The Journal of Applied Business Research, vol. 18, n. 1, 2002, p. 61-77. 
AMIRI, Arshia; GERDTHAM, Ulf-G. Granger Causality Between Exports, Imports and GDP in France: Evidence from Using Geostatistical Models. The Economic Research Guardian, vol. 2, n. 1, 2012, p. 43-59.

BALZANA FILHO, Marcelo de Lima; BORDEAUX-REGO, Ricardo. Uma Análise da Relação Entre o Retorno das Ações do Setor de Construção Civil Brasileiro e Indicadores Macroeconômicos. Engevista, V.16, n.2, p.137-151, junho 2014.

BERTELLA, Mário A.; SILVA, Roseli; PEREIRA, Renan A.M. Cointegração e Causalidade entre Indicadores Macroeconômicos e Índice Bovespa. Apresentação em Seminário, 2009.

BONE, Rosemarie B. Existe causalidade entre as ações da Petrobrás Holding e o Ibovespa no período de 1994-2002?. $2^{\circ}$ Congresso Brasileiro de P\&D em Petróleo e Gás.

BOUCHER, Chistophe. Stock Prices, Inflation and Stock Returns Predicability. Revue de l'association française de finance, vol. 27, n. 2/2006, p 71-101.

BUSSAB, Wilton O.; MORETTIN, Pedro A. Estatística Básica. $5^{\circ}$ edição, Editora Saraiva, 2004.

CAMPBELL, John $\quad$ Y.; VUOLTEENAHO, Tuomo. Inflation Illusion and Stock Prices. The American Economic Review, vol. 94, maio de 2004, p 19-23.

CARNEIRO, Francisco G. A Metodologia dos Testes de Causalidade em Economia. Brasília, Universidade de Brasília, Série Textos Didáticos, n. 20, 1997.

CASTRO, Marcos V.S.C. Uma análise empírica da relação entre inflação e retornos do mercado acionário brasileiro. Dissertação de Mestrado, Rio de Janeiro, Instituto Brasileiro de Mercado de Capitais, 2009.

DAVIDSON, R. e MACKINNON, J.G. Estimation and Inference in Econometrics. Oxford University Press, 1993.

DEPARTAMENTO INTERSINDICAL DE ESTATÍSTICA E ESTUDOS SOCIOECONÔMICOS. Estudos e Pesquisas: Estudo Setorial da Construção. Em http://www.dieese.org.br/esp/estudos_set oriais.xml, n.56, abril de 2011.

DICKEY, David A.; FULLER, Wayne A. Distribution of the Estimators for Autoregressive Time Series With a Unit Root. Journal of the American Statistical Association, vol. 74, n. 366, junho de 1979, p. 427-431.

FAMA, Eugene F. Stock Returns, Real Activity, Inflation, and Money. The American Economic Review, vol. 71, n. 4, setembro de 1981, p. 545-565.

GAN, Christopher; LEE, Minsoo; YONG, Hua H. A.; ZHANG, Jun. Macroeconomic Variables and Stock Market Interactions: New Zealand Evidence. Investment Management and Financial Innovations, vol. 3, n. 4, 2006, p. 89-101.

GESKE, Robert; ROLL, Richard. The Fiscal and Monetary Linkage Between Stock Returns and Inflation. The Journal of Finance, vol. 38, n. 1, março de 1983, p 1-33.

GJERDE, Øystein; SÆTTEM, Frode. Causal relations among stock returns and macroeconomic variables in a small, open economy. Journal of International Financial Markets, Institutions and Money, vol. 9, n. 1, 1999, p 61-74.

GRANGER, C. W. J. Investigating Causal Relations by Econometric Models and Cross-spectral Methods. 
Econometrica, vol. 37, n. 3, agosto de 1969, p 424-438.

GRÔPPO, Gustavo S. Causalidade das variáveis macroeconômicas sobre o IBOVESPA. Dissertação de Mestrado, Piracicaba, Universidade de São Paulo, 2004.

GULTEKIN, Bulent. Stock Market Returns and Inflation: Evidence from Other Countries. The Journal of Finance, vol. 38, n. 1, março de 1983, p. 49-65.

KISAKA, Sifunjo E.; MWASARU, Anthony. The Causal Relationship between Exchange Rates and Stock Prices in Kenya. Research Journal of Finance and Accounting, vol. 3, n. 7, 2012, p. 121-130.

LAWAL, Nurudeen A.; OKUNOLA, Oluniyi E. Stock Prices, Stock Market Operations and Nigerian Economic Growth: A Granger Causality Modelling. Global Advanced Research Journal of Management and Business Studies, vol. 1, n. 10, novembro de 2012, p. 375-383.

LEE, Bong-Soo. Causal Relations Among Stock Returns, Interest Rates, Real Activity, and Inflation. The Journal of Finance, vol. 47, n. 4, setembro de 1992, p. 1591-1603.

MADDALA, G.S. Introduction to Econometrics. Wiley, 2001.

MAHMOOD, Wan M.W.; DINNIAH, Nazihah M. Stock Returns and Macroeconomics Variables: Evidence from the Six-Asian Pacific Countries. International Research Journal of Finance and Economics, n. 30, 2009, p. 154-164.

MARGARIDO, Mario A.; ANEFALOS, Lilian $C$. Testes de raíz unitária e o software SAS. Agricultura em São Paulo, vol. 46, n. 2, 1999, p. 19-45.

MATOS, Orlando C. Desenvolvimento do Sistema Financeiro e Crescimento Econômico no Brasil: Evidências de
Causalidade. Trabalhos para Discussão, Brasília, n. 49, setembro de 2002, p. 1-64.

MD-YUSUF, Mazila; RAHMAN, Hamisah Abd. Granger Causality Relationship between Malaysia Equity Market and Exchange Rate Volatility. International Journal of Trade, Economics and Finance, vol. 4, n. 1, fevereiro de 2013, p. 19-24.

NUNES, Maurício; COSTA Jr, Newton C. A.; MEURER, Roberto. A Relação entre o Mercado de Ações e as Variáveis Macroeconômicas: Uma Análise Econométrica para o Brasil. Revista Brasileira de Economia, vol. 59, n. 4, out/dez de 2005, p 585-607.

PERALES, Norma A. H.; ROBINS, Russel. The Relationships between Mexican Stock Market Returns and Real, Monetary and Economic Variables. Disponível em: $<$ http://egade.sistema.itesm.mx/investigac ion/documentos/documentos/13egade_no rmahdz.pdf>, 2002.

RAY, Sarbapriya. E Testing Granger Causal Relationship between Macroeconomic Variables and Stock Price Behaviour: Evidence from Índia. Advances in Applied Economics and Finance (AAEF), vol. 3, n. 1, 2012, p. 470-481.

RIBAS, José R.; VIEIRA, Paulo R.C. Análise Multivariada com o uso do SPSS. $5^{\circ}$ edição, Rio de Janeiro, Editora Ciência Moderna, 2011.

SINGH, Dharmendra. Causal Relationship Between MacroEconomic Variables and Stock Market: A Case Study for India. Pakistan Journal of Social Sciences, vol. 30, n. 2, dezembro 2010, p. 263-274.

SUM, Vichet. Impulse Response Functions and Causality Test of Financial Stress and Stock Market Risk Premiums. Revue de l'association française de finance, vol. 4, n. 1, 2013, p. $1-4$. 